\title{
Obstacle Avoidance System for a Quadrotor UAV
}

Joseph Wagster, Matthew Rose, and Hovig Yaralian

California State Polytechnic University, Pomona, CA 91768

This is the summary of the research being done at Cal Poly Pomona at an undergraduate level on the development of obstacle avoidance capability for unmanned aerial vehicles (UAVs). A quadrotor $\mathrm{UAV}$ is used as the research platform. Push and Rapidly Exploring Random Tree (RRT) algorithms are used for the obstacle avoidance capability. The paper shows the use of cheaper commercial off-the-shelf sensors and processor board in the design and implementation of obstacle avoidance algorithm.

\section{Nomencalture}

$$
\begin{array}{ll}
\mathrm{a}_{\mathrm{x}} & =\text { actual forward acceleration }\left(\mathrm{ft} / \mathrm{sec}^{2}\right) \\
\mathrm{a}_{\mathrm{y}} & =\text { actual side acceleration }\left(\mathrm{ft} / \mathrm{sec}^{2}\right) \\
\mathrm{a}_{\mathrm{z}} & =\text { actual vertical acceleration }\left(\mathrm{ft} / \mathrm{sec}^{2}\right) \\
\mathrm{g} & =\text { acceleration due to gravity }\left(\mathrm{ft} / \mathrm{sec}^{2}\right) \\
\mathrm{m} & =\text { quadrotor mass }(\mathrm{slug}) \\
\mathrm{p} & =\text { roll rate }(\mathrm{rad} / \mathrm{sec}) \\
\mathrm{q} & =\text { pitch rate }(\mathrm{rad} / \mathrm{sec}) \\
\mathrm{r} & =\text { yaw rate }(\mathrm{rad} / \mathrm{sec}) \\
\mathrm{T} & =\text { engine thrust }(\mathrm{b}) \\
\mathrm{u} & =\text { forward velocity }(\mathrm{ft} / \mathrm{sec}) \\
\mathrm{v} & =\text { side velocity }(\mathrm{ft} / \mathrm{sec}) \\
\mathrm{w} & =\text { vertical velocity }(\mathrm{ft} / \mathrm{sec}) \\
\dot{\mathrm{u}} & =\text { measured forward acceleration }\left(\mathrm{ft} / \mathrm{sec}^{2}\right) \\
\dot{\mathrm{w}} & =\text { measured side acceleration }\left(\mathrm{ft} / \mathrm{sec}^{2}\right) \\
\dot{\mathrm{v}} & =\text { measured vertical acceleration }\left(\mathrm{ft} / \mathrm{sec}^{2}\right) \\
\mathrm{X} & =\text { longitudinal force }(\mathrm{lb}) \\
&
\end{array}
$$




$$
\begin{array}{ll}
\mathrm{Y} & =\text { side force }(\mathrm{lb}) \\
\mathrm{Z} & =\text { vertical force }(\mathrm{lb})
\end{array}
$$

\section{Motivation and Project Goals}

Research pertaining to Unmanned Aerial Vehicles (UAVs) continues to increase due to the versatility of their potential use for both military and humanitarian missions such as search and rescue, surveillance of disaster stricken areas, and battlefield assessment. However small UAVs are typically not capable of obstacle avoidance due to the heavy, expensive, and energy consuming nature of the sensors necessary for detection and analysis of obstacles. Despite these many applications, the use of UAVs is limited to military and restricted airspaces due to the lack of such technology on small UAVs makes them vulnerable to incoming objects such as other vehicles as well as stationary obstacles such as walls or buildings. For cost effective use of these vehicle in the National Airspace System, the Federal Aviation Administration is requiring that UAVs be equipped with collision and obstacle avoidance systems.

Due to importance of the topic, many organizations and universities are conducting research on obstacle avoidance capability for the UAVs [1-3]. Recently, the research community has shown increased interest in developing the obstacle avoidance capability for quadrotors [4]. A quadrotor has the ability to hover and is capable of instantaneously changing direction, eliminating the need to incorporate minimum radius turning into the algorithm. Potential military application includes the use of such vehicles for reconnaissance inside unfamiliar buildings for situational awareness before sending troops in. In a similar scenario, vehicles with obstacle avoidance could allow for search and rescue teams to find survivors in buildings damaged by a disaster without the risk of injuring the rescuers. While small cameras may be mounted to the vehicle in such scenarios they do not give a complete field of view which in the event of a forward facing camera could result in loss of the vehicle if an object is behind the vehicle as it attempts to back up.

Cal Poly Pomona has recently started research on the development of obstacle avoidance capability for a quadrotor UAV. The goal of this research was to develop the capability using light weight, inexpensive, and low energy consuming components with the potential to map and navigate unfamiliar environments. The following sections talk about the hardware selection, approach used in the research, results, conclusion, and future work. 


\section{Hardware}

An attempt to maximize the use of Commercial-Off-The-Shelf (COTS) components was made to ensure repeatability and cost effectiveness of the research solution. A trade study, as shown in Table 2.1, was done to determine the best autopilot for this research. Low cost and memory size were the highest weighted categories in order to minimize overall system cost and maximize potential to perform onboard mapping.

Table 2.1: Autopilot Trade Study

\begin{tabular}{|c|c|c|c|c|c|}
\hline Autopilot & Cost & Memory & Tech Support & Programming & Assembly \\
\hline ArduPilot & $\sim \$ 300$ & Ok & Limited & Simple & Simple \\
\hline Lisa-L & $\sim \$ 700$ & Expandable & Active & Complex & Complex \\
\hline MicroPilot & $\sim \$ 3000$ & N/A & Company & N/A & Simple \\
\hline Piccolo II & $\sim 5000$ & N/A & Company & N/A & Simple \\
\hline
\end{tabular}

The Lisa-L autopilot by Paparazzi was chosen for its superior memory capabilities [5]. However, a wiring issue resulting in a short resulted in discovery that the autopilot was severely back ordered once the project was started. It was later decided to use the ArduPilot Mega autopilot by DIY Drones [6].

A sensor trade study was conducted to determine the most effective sensor for use in the research as shown in Table 2.2. The primary weighting factors for this trade were cost and weight as the sensors needed to comply with the goal of a light weight and inexpensive system. Max Sonar-EZ0 [7] was chosen due to their low mass, energy consumption, and cost.

Table 2.2: Sensor Trade Study

\begin{tabular}{|c|c|c|c|c|}
\hline Sens or & Range $(\mathbf{m})$ & Mass $(\mathrm{g})$ & Cost $(\$)$ & Energy Cons umption $(\mathbf{m A})$ \\
\hline Max Sonar-EZ0 & $0-1$ & 4.3 & 30 & 2 \\
\hline SICK Lidar & $0-10$ & 1200 & 4,000 & 470 \\
\hline Xbox Connect & $0-2$ & 5606 & 99 & 5 \\
\hline
\end{tabular}

Six sonar sensors were mounted to a custom built frame to produce a quadrotor capable of determining distances to objects above, below, left, right, forward, and rear of the vehicle. The autopilot was mounted to a carbon plate in the middle and a carbon rod was bent around the aircraft to ensure the props would not break if the vehicle hit an obstacle. Figure 2.1 shows the completed vehicle. 


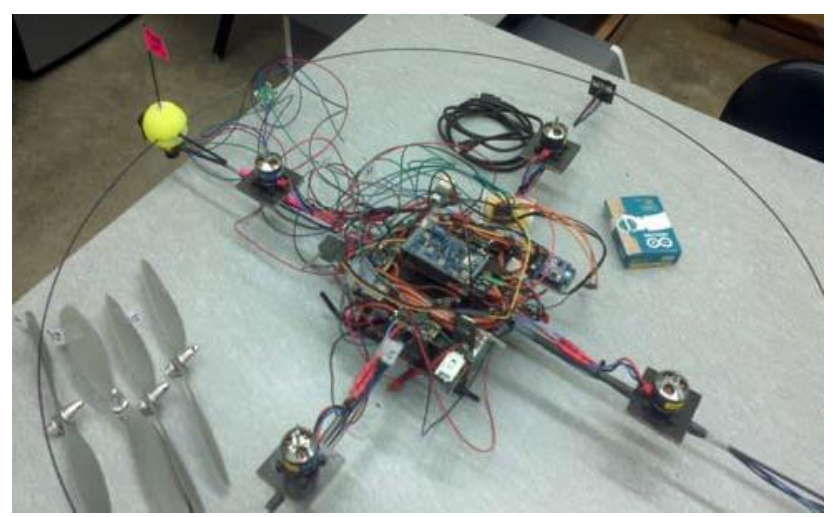

Figure 2.1: Completed Quadrotor

\section{Approach}

Overall system avoidance and mapping was broken down into three levels of avoidance to minimize risk to the vehicle as well as ensure extraction of useful information from the research in the event the final goal was not reached.

The first level of avoidance utilizes a forward facing sensor to determine presence of and distance to obstacles in front of the vehicle. A tolerance is set for the minimum acceptable distance for objects in front of the UAV such that the vehicle will not collide with the wall before it is capable of ceasing forward motion of the vehicle. This tolerance is used within an algorithm that initiates a pitch angle that causes the vehicle to slow down and return to a safe distance away from the obstacle.

Level two avoidance utilizes sensors to the left and right of the vehicle in order to center the vehicle between two obstacles using push algorithm. The algorithm for this method involves taking the sensor distance and converting it to a roll angle that increases as the vehicle gets closer to the object. Effective roll angles relative to the distance from the object were to be determined from effectiveness of avoidance at various distances in level one avoidance testing. Determining the commanded roll necessary to keep the vehicle equidistant from

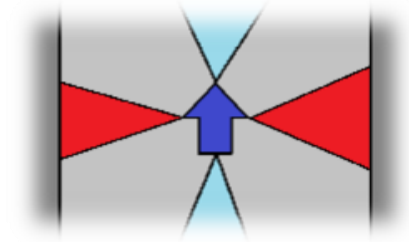

Figure 3.1: Level Two Avoidance two objects on either side of the vehicle was done by associating a positive roll contribution from the sensor on one side and negative roll contribution from the sensor on the other side as shown in Figure 3.1.

Minimization of large roll angles when the vehicle is near the center of two objects on either side of the vehicle is obtained by having a small change in roll angle for a given change in distance if the vehicle is far from 
the obstacle and large change in roll angle for a given change in distance if the vehicle is near the obstacle as shown in Figure 3.2.

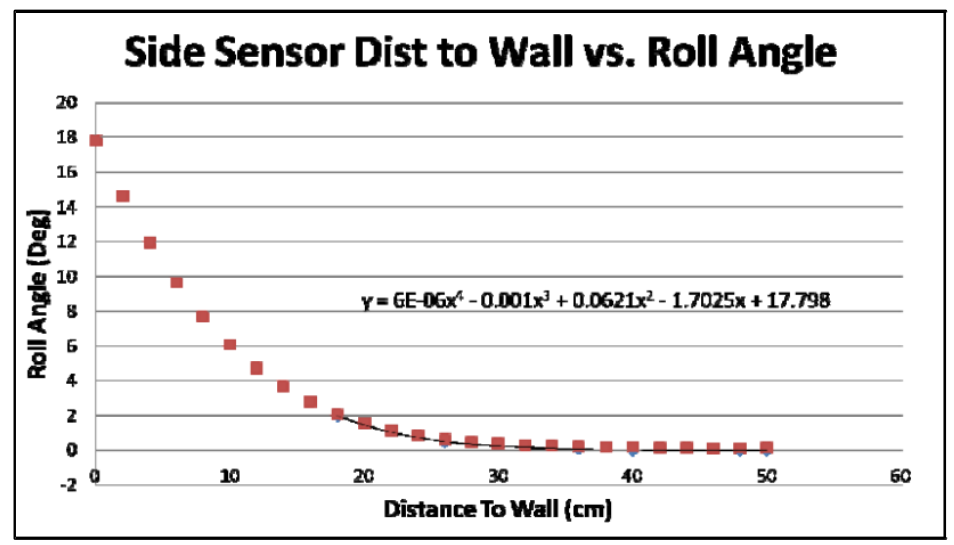

Figure 3.2: Roll Angle of Quadrotor Due to Sensor Input

The flight test designed to test this algorithm involved flying the quadrotor down a hallway where the vehicle would constantly have an obstacle, a wall, on either side. With the algorithm working correctly the quadrotor may initially start closer to one wall and roll to its left and right, guided by the distance to each wall, until it settles in the middle. Initial testing of this algorithm is expected to result in an under damped system where the vehicle oscillates from left to right. However, through modification of roll angle per change in distance to the wall, higher damping of the system is possible.

It was determined that a modified Rapidly Exploring Random Trees algorithm [8] was optimum for use in obstacle avoidance as it not only avoids the obstacle but also plans a path around it. In implementing this algorithm, the quadrotor determines its flight path based on point value scores of its available movements. The quadrotor is limited to incremental forward, backward, left, and right movements as shown in Figure 3.3. A score for each target position is calculated by adding a score of $1,000,000$ to any direction for which the sonar picks up an obstacle in addition to adding one point

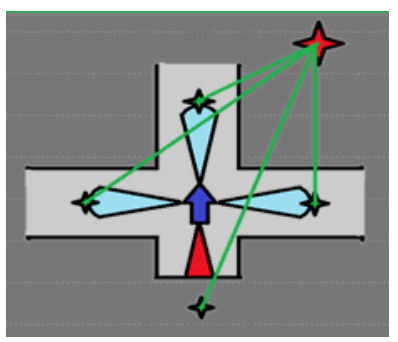

Figure 3.3: Point Based Path for each meter in distance between the next waypoint and the target waypoint. The quadrotor can choose the waypoint that is closest to the target by choosing the one with the smallest score.

Free body diagrams were used in determining quadrotor accelerations. Figure 3.4 shows the forces and angles associated with rolling and pitching motions of the quadrotor. If engines one and two are rotating at certain equal 
speeds and engines three and four are both rotating at the same speed that is different from the speed of engines one and two, the quadrotor will yaw to one direction.

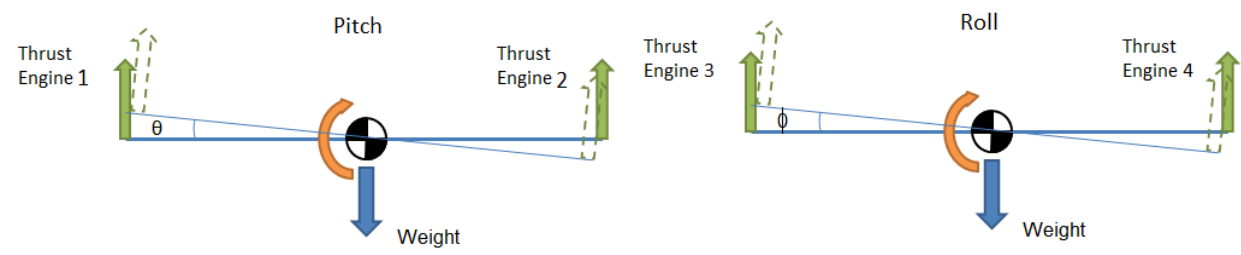

Figure 3.4: Side Vie w Free Body Diagram of the Quadrotor

Using the free body diagrams and a rigid body approximation, the relationship between each engine's thrust, quadrotor pitch and roll angles, and the quadrotor's accelerations were determined as given below.

$$
\begin{aligned}
& a_{x}=\frac{T_{8} \sin \theta+T_{4} \sin \theta+T_{8} \cos \psi+T_{4} \cos \psi}{m} \\
& a_{y}=\frac{T_{1} \sin \phi+T_{2} \sin \phi+T_{3} \sin \psi+T_{4} \sin \psi}{m} \\
& a_{z}=\frac{T_{3} \cos \theta+T_{4} \cos \theta+T_{1} \cos \phi+T_{2} \cos \phi}{m}
\end{aligned}
$$

The accelerations are then found using the attitude angles determined by the inertial measurement unit and the thrust determined from the look up tables that were generated from the bench testing. From the dynamics equations of rigid body aircraft [9], the forces in the body-axis system are given by the following equations: mea

$$
\begin{aligned}
& X-m g \sin (\theta)=m(\dot{u}+q w-r v) \\
& Y+m g \cos (\theta) \sin (\Phi)=m(\dot{v}+r u-p w) \\
& Z+m g \cos (\theta) \sin (\Phi)=m(\dot{w}+p v-q u)
\end{aligned}
$$

By dividing equations 4-6 by mass and using small angle approximations, equations relating measured accelerations, actual accelerations, and velocity are obtained as given below [10].

$\mathrm{Me}$

$$
\begin{aligned}
& \dot{u}=a_{x}-q w+r v-g \theta \\
& \dot{v}=a_{y}-r u+p w+g \Phi \\
& \dot{w}=a_{z}-p v+q u-g \theta_{0} \theta
\end{aligned}
$$

Using equations 7-9, the measured accelerations in addition to the calculated accelerations can be used to 
determine the quadrotor's velocity. This velocity is then integrated to determine actual position of the quadrotor. 


\section{Results}

Bench testing of the sonar through the autopilot was done to calibrate the sensors such that they gave the autopilot accurate distance to the obstacles they detected. Sonar data taken from each of the six sensors mounted on the vehicle was compared to actual distances from the sensors, both individually and simultaneously. After calibration of the sensors, a flight test of level one avoidance was conducted with a tolerance distance of 0.3 meters and a blow tolerance pitch back angle of five degrees. The quadrotor was sent toward the obstacle at approximately one foot per second and upon encountering the obstacle it successfully pitched back five degrees and did not run into the obstacle. The response of the vehicle
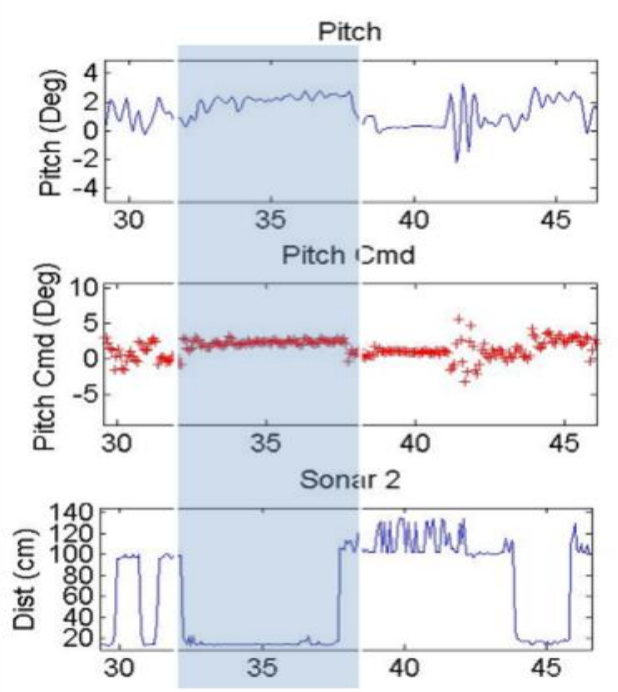

Figure 4.1: Pitch Back Command When Minimum Distance Triggered was slow and it was not advisable to attempt the test at higher speeds. The data compiled from the flight test depicted in Figure 4.1 shows that while the sonar sensor was blocked by the obstacle, a commanded pitch angle of five degrees back was successfully given to the autopilot.

Level two bench testing was conducted by blocking one of the sonar while leaving the other sonar unblocked. The data from this test shows that the while a sonar is blocked, an erratic roll and pitch command is issue. The erratic behavior of the commanded angles is due to fluctuation in distance reported by sonar two as a result of not creating a case ignoring impossible distances or ignoring distances recorded while the reported distance is fluctuating due to erroneous readings at maximum distance as shown in Figure 4.2.
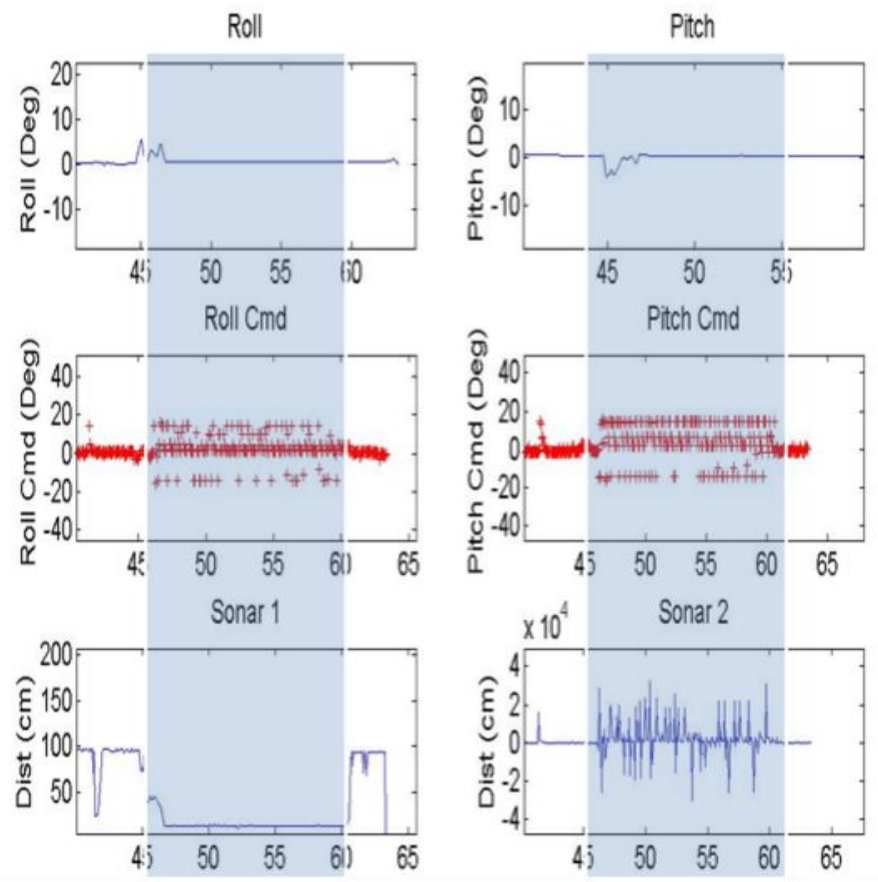

Figure 4.2: Level Two Avoidance Bench Test Results 


\section{Conclusions and Future Work}

Level one avoidance was successful, however longer range sensors are necessary for faster flight as the obstacles would need to be detected sooner. Additionally, flight testing of level one avoidance showed that the sensors would either need to be gimbaled or an algorithm would have to be implemented to determine distance to objects as the quadrotor tilted. Lag in sensor data output through serial showed that the system would need more processing power if onboard path planning were to be implemented. The future work for this research includes completion of level two avoidance bench and flight testing as well as implementation of the point based algorithm and investigation of longer ranged sensors.

\section{References}

[1] Utt, J., McCalmont, J., et al, "Development of Sense and Avoid System”, AIAA Infotech@Aerospace Conference, Arlington Virginia, September 26-29, 2005.

[2] He, Z., Iyer, R. V., et al, "Vision-Based UAV Flight Control and Obstacle Avoidance", Proceedings of American Control Conference, Minneapolis, Minnesota, June, 14-16, 2006.

[3] Martel, F., Schultz, R. R., et al, 'Unmanned Aircraft Systems Sense and Avoid Avionics utilizing ADS-B Transceiver”,AIAA Infotech@Aerospace Conference, Seattle, Washington, April 6-9, 2009.

[4] Bouadallah, S., Becker, M., et al, "Toward Obstacle Avoidance on Quadrotors", Proceedings of the XII International Symposiumon Dynamic Problems of Mechanics, Ilhabela, SP, Brazil, Feb 26-March 2, 2007.

[5] Paparazzi, http://paparazzi.enac.fr/wiki/Main_Page.

[6] DIY Drones, "Arducopter", http://code.google.com/p/arducopter/wiki/ArduCopter.

[7] Max Botix, "Sonar Range Finder", http:/www.maxbotix.com/documents/MB1000_Datasheet.pdf.

[8] Call, R., Brandon, “Obstacle Avoidance for Unmanned Aerial Vehicles", Thesis for Bringham Young University, Idaho, United States, December 2006.

[9] Nelson, C., Robert, Flight Stability and Automatic Control, $2^{\text {nd }}$ Ed., McGraw-Hill, United States, 1998.

[10] Cheng, P., Rendy, Tischler, B., Mark, Schulein, J., Greg, "R-MAX Helicopter State-Space Model Identification for Hover and Forward-Flight”, United States, April 2006. 